\title{
An integrated optical scheme for interrogation of interferometric fiber optic sensors
}

\author{
A.S. Kozlov, I.V. Ilichev, and A.V. Shamray \\ loffe Physico-Technical Institute \\ Polytekhnicheskaya str, St. Petersburg, 194021, Russia \\ e-mail: achamrai@mail.ioffe.ru
}

\section{Introduction}

Fiber optical interferometers are widely used as the sensing elements for measurements of temperature, electric and magnetic fields, acoustic and seismic waves, applied strain etc, because of its high sensitivity, large dynamic range, and convenience for multiplexing [1]. The output of an interferometer is a cosine function of the phase difference between two arms. The phase difference drifts because of the low frequency random temperature and pressure fluctuations, which interferometer arms experience. The drift causes random changes in the detected signal, which are called signal fading. To reduce signal fading several demodulation schemes have been developed [2].

The homodyne detection scheme using phase generated carrier (PGC) exhibits high efficiency, and good linearity, that makes this technique very attractive. In the PGC demodulation scheme, a highfrequency carrier is required to modulate an interferometer. So the key element in this approach is an optical phase modulator for generation of the high frequency carrier.

We have developed and fabricated a multifunctional integrated optical scheme (MIOS), which can be used in the PGC demodulation system. The scheme includes three optical components on the one substrate (Fig.1): the optical polarizer, X-coupler, and electrooptical phase modulator.

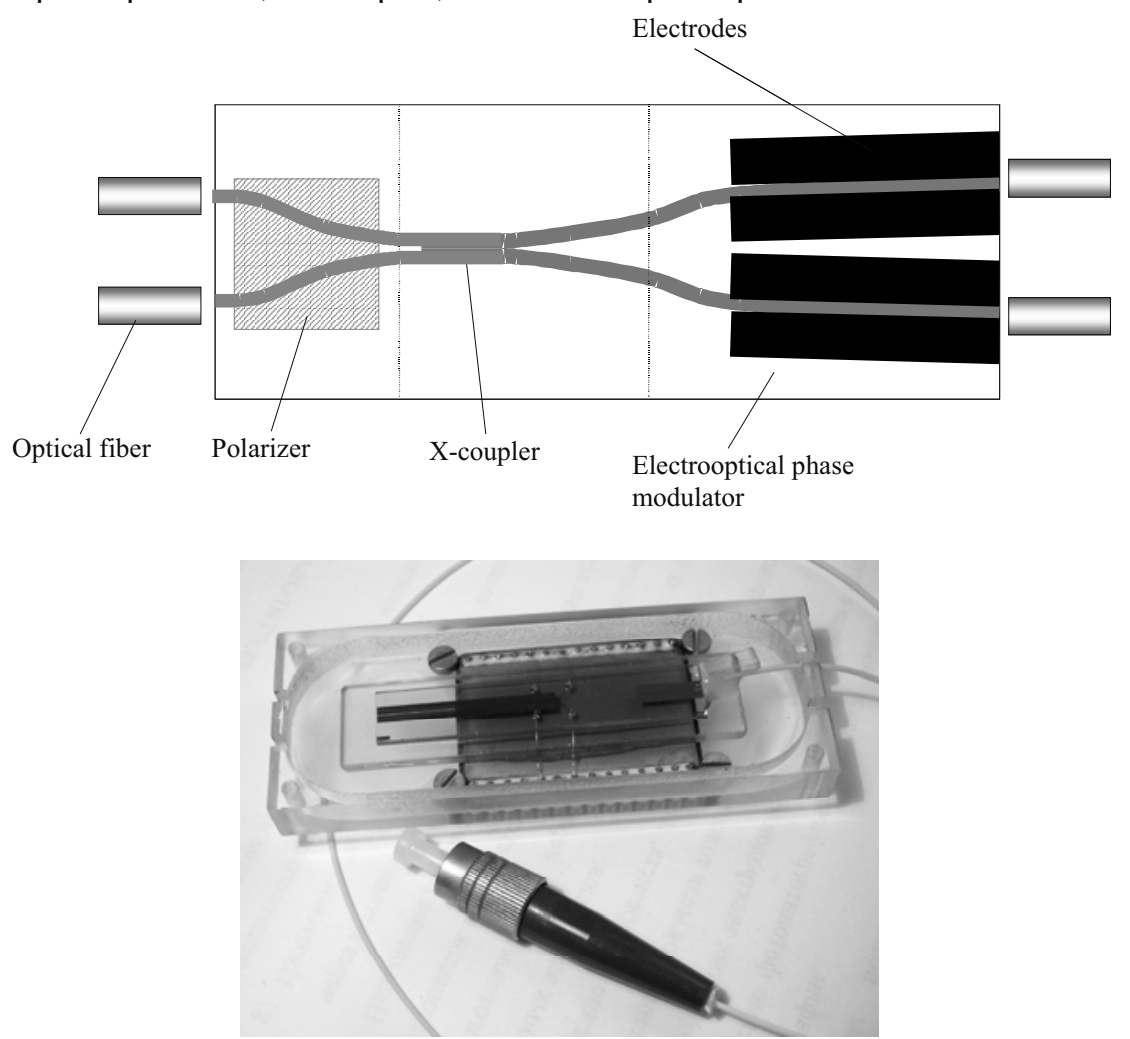

Fig. 1. Multifunctional integrated optical scheme (MIOS).

\section{Device fabrication}

The MIOS was fabricated on the $\mathrm{LiNbO}_{3}$ substrate by the conventional method of Ti thermodiffusion [3]. It comprises single-mode channel optical waveguides for the light in $1500-1600 \mathrm{~nm}$ wavelength range. A special topology of the photo-mask has been developed and technological conditions for waveguide fabrication have been elaborated. The aim was to obtain simultaneously waveguides with good coupling efficiency from standard telecommunication single mode fiber (SMF-28) and X-coupler with exact 3dB 
splitting ratio. The integrated optical scheme with total optical losses (fiber to fiber) as low as $2.5 \mathrm{~dB}$ was produced. A special trimming procedure based on a high temperature annealing was used for adjustment of the splitting ration of $X$-coupler with accuracy of $1 \%$. High accuracy of the splitting ratio provides high contrast of a signal modulation.

The optical polarizer was produced by deposition on the waveguides of a thin metal film with a dielectric buffer layer. The principle of operation of the polarizer is based on the efficient coupling of TM mode of the optical waveguide to the damping plasmon-polariton mode of the thin metal film, and the dielectric buffer layer is used for the phase matching [4]. We have used $\mathrm{Al}$ metal film and $\mathrm{Al}_{2} \mathrm{O}_{3}$ dielectric buffer layer. This combination of materials is very convenient since it can be produced in one technological stage with the using of reactive sputtering. The optimal thicknesses of the metal film and the dielectric buffer layer have been determined. The polarizer with very high extinction ratio (more than 45 $\mathrm{dB}$ ) has been fabricated.

We used standard $\mathrm{Au} / \mathrm{Cr}$ electrodes for application of an external electric field for the electrooptical phase modulation. Since relatively low frequencies $(<1 \mathrm{MHz})$ were used for a signal modulation, there were no special requirements to electrodes configuration and impedance. The geometry of the transverse electrooptical effect and short distance between electrodes ensured low halfwave voltage (lower than $10 \mathrm{~V}$ ).

\section{New interrogation technique}

The integrated optical scheme of a similar configuration is used in high accuracy fiber optical gyroscopes for demodulation of a signal from Sagnac interferometer [5]. In this work an original algorithm has been developed to use MIOS in the PGC demodulation system, which can be applied for the interrogation of Mach-Zehnder, Michelson, Fabry-Perot and others fiber optical interferometers.

The distinguishing feature of the proposed technique is the rectangular two-level high frequency modulation signal. To provide a rectangular phase modulation with sharp edges an integrated electrooptical modulator with a broad bandwidth is used. The levels of modulation signal are corresponding to the points with maximum slope on the interferometer transfer function. If the interferometer has a zero optical path difference, the output signal at the frequency of modulation equals to zero (Fig. 2). When some phase difference appears due to external influence on the interferometer, the output signal is proportional to this phase difference or shift of the interferometer transfer function. The phase of the output signal provides information about sign of the phase difference between two arms of the interferometer. This signal can be easily detected using digital lock-in technique. The output signal can be used as an error signal of a feedback control system, which provides a low frequency signal on the electrooptical modulator to compensate the phase difference in the interferometer.

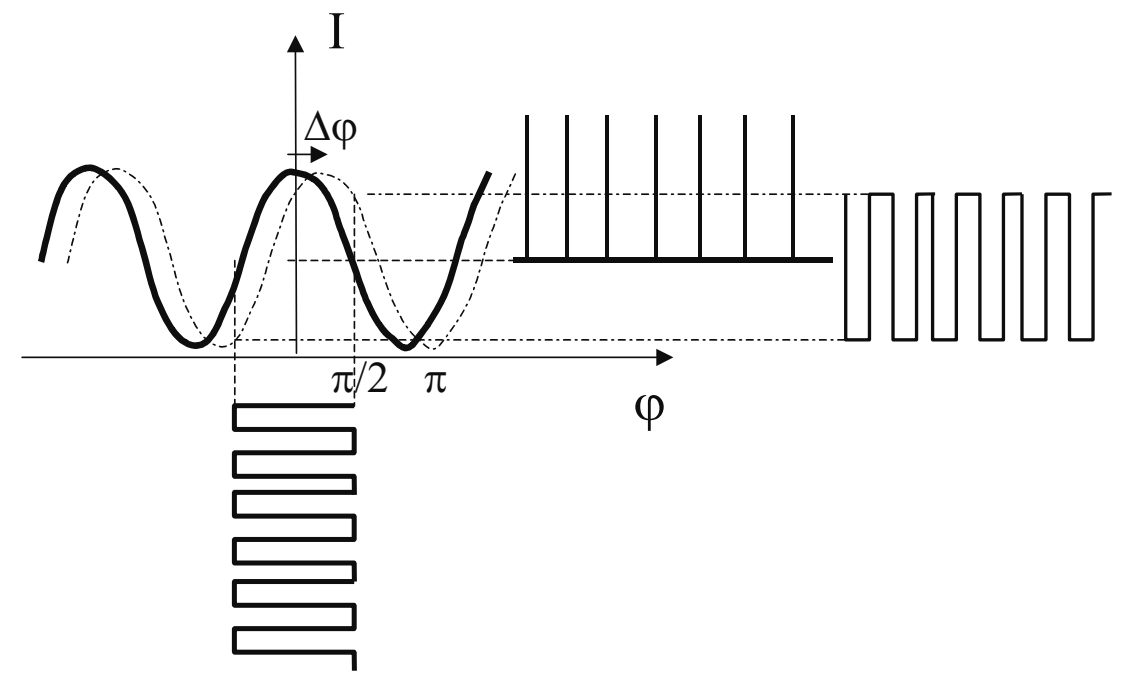

Fig. 2. The algorithm of interrogation of a fiber optical interferometer.

This low frequency feedback signal is a detected signal of the interferometric fiber optic sensor. Since the electronic scheme works with signals of very small amplitude, high gain coefficient can be used. Moreover digital electronics is used for the signal processing. Thus the system has a high linearity, very high sensitivity and huge dynamic range, which are determined by characteristics of digital-to-analog and analog-to-digital converters. The system can be used for interrogation sensors based on the interferometers of different types. It also can be adopted for a broadband incoherent light source. And the 
using of the modulation signal with several (more than two) levels will provide additional functionality for signal processing.

To demonstrate of the efficacy of the proposed technique a prototype of the system has been used for interrogation of interferometric displacement sensor (Fig.3). Four level modulation (total amplitude equal to $2 \pi$ ) provided addition functionality such as determination of the sign of the feedback signal and self-tuning of the modulation amplitude. The accuracy of $1 \mathrm{~nm}$ for the displacements of several millimeters has been easily obtained.

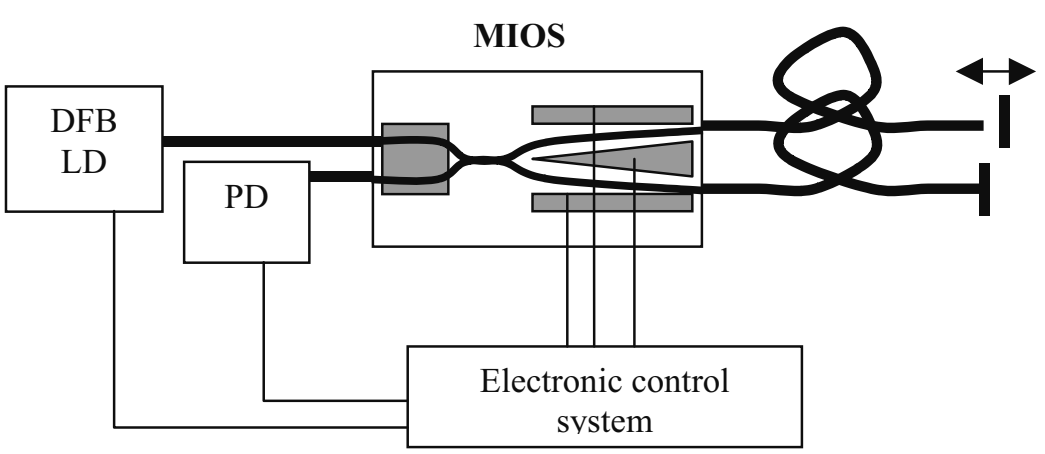

Fig. 3. Interferometric displacement sensor with new interrogation algorithm

\section{Conclusion}

New interrogation technique for interferometric fiber optic sensors has been developed. This technique exploits PGC demodulation approach with a phase modulation by signal of a rectangular shape. In this case digital electronics can be used for the signal processing, and the system has a high linearity, very high sensitivity and huge dynamic range, which are determined by characteristics of digital-to-analog and analog-to-digital converters. A multifunctional integrated optical scheme for experimental realization of the proposed technique has been developed and fabricated. The experimental demonstration of the interrogation of an interferometric displacement sensor has been performed.

\section{References}

1. T.G. Giallorenzy, J.A. Bucaro, A. Dandridge, G.H. Sigel, J.H. Cole, S.C. Rashleigh, and R.G. Priest, "Optical fiber sensor technology," IEEE J. Quantum. Electron., vol. QE-18, pp. 626-666, 1982.

2. $\quad$ C. K. Kirkendall, A. Dandridge, "Overview of high performance fibre-optic sensing," J. Phys. D: Appl. Phys., vol. 37, pp. R197-R216, 2004.

3. M. Lawrence, "Lithium niobate integrated optics," Rep. Prog. Phys., pp.363 - 429, 1993.

4. Yu.N. Konoplev, Yu.A. Mamaev, and A.A. Turkin, @Optimization of the structure of an optical fiber polarizer with a metal film at a wavelength of $980 \mathrm{~nm}$," Optics and Spectroscopy, vol. 105, pp. 128-133, 2008.

5. H.C. Lefevre, S Vatoux, M. Papuchon, C. Puech, "Integrated: optics: a practical solution for the fiber-optic gyroscope," Proc. SPIE, vol. 719, pp. 101-112, 1986. 